\title{
Investigation into the potential of GAGG:Ce as a neutron detector
}

\author{
M. P. Taggart ${ }^{\mathrm{a}, *}$, M. Nakhostin ${ }^{\mathrm{a}}$, P. J. Sellin ${ }^{\mathrm{a}}$ \\ ${ }^{a}$ Department of Physics, University of Surrey, Guildford, Surrey, U.K.
}

\begin{abstract}
In this work we investigate the potential use as a thermal neutron detector of cerium-doped gadolinium aluminium gallium garnet (GAGG:Ce) coupled to a silicon photomultiplier ( $\mathrm{SiPM})$. The response to thermal neutrons has been measured, with two strong low energy neutron-indicative peaks clearly identifiable below $100 \mathrm{keV}$ and additional $\gamma$ peaks at higher energies. The neutron-related peaks are produced by a combination of contributions from excited states of the two isotopes ${ }^{156} \mathrm{Gd}$ and ${ }^{158} \mathrm{Gd}$ which can be clearly resolved in our GAGG scintillation detector. In particular, two peaks due to neutron-induced $\gamma$-ray emission are observed at approximately $82 \mathrm{keV}$ and $260 \mathrm{keV}$, with best achieved energy resolutions of $24.1 \pm 0.2 \%$ and $22.7 \pm 0.7 \%$ respectively. Three different scintillator volumes $\left(0.1 \mathrm{~cm}^{3}, 0.4 \mathrm{~cm}^{3}\right.$, and $\left.1 \mathrm{~cm}^{3}\right)$ were investigated and the respective results for each configuration will be presented.

Our findings show that a GAGG-SiPM based detector can be used as a compact, efficient thermal neutron detector in a low $\gamma$-ray contamination environment.
\end{abstract}

Keywords: Neutron detection, GAGG:Ce, silicon photomultiplier.

\section{Introduction}

Cerium-doped gadolinium aluminium gallium garnet (GAGG:Ce - $\mathrm{Gd}_{3} \mathrm{Al}_{2} \mathrm{Ga}_{3} \mathrm{O}_{12}$ ) is a relatively new scintillator showing good promise as the potential detector

\footnotetext{
* Corresponding author

Email address: m.taggart@surrey.ac.uk (M. P. Taggart)
}

Preprint submitted to Nuclear Inst. ES Methods A

May 7, 2019 
Table 1: Typical values of GAGG:Ce characteristics compared to common alternate PET scintillators 1,5 .

\begin{tabular}{c||c|c|c}
\hline & GAGG:Ce & BGO & LYSO:Ce \\
\hline Light Yield (photons/MeV) & $40-55000$ & $8-10000$ & $\sim 32000$ \\
$\lambda_{\text {peak }}(\mathrm{nm})$ & 520 & 480 & 420 \\
Density $\left(\mathrm{g} / \mathrm{cm}^{3}\right)$ & 6.63 & 7.13 & 7.1 \\
$\mathrm{Z}_{\text {eff }}$ & 54 & 74 & 66 \\
Energy Resolution (\%) & $\sim 5.2$ & $\sim 9-15$ & $\sim 7.9$ \\
\hline
\end{tabular}

of choice for PET 1, 2] and SPECT [3, 4] applications. GAGG has many char5 acteristics making it highly suitable for use as a $\gamma$-ray detector, particularly for a PET system. It is one of the brightest scintillators available today with a light yield of 44,000 photons/MeV which is slightly greater than that of $\mathrm{NaI}(\mathrm{Tl})$ $(38,000$ photons/MeV). It has a density approaching the levels of BGO and LYSO $\left(6.63 \mathrm{~g} / \mathrm{cm}^{3}\right)$, and a gadolinium mass fraction of $\sim 50.8 \%$ resulting in an effective atomic number of 54 .

Furthermore, with an energy resolution of $\sim 5 \%$ at $662 \mathrm{keV}$, equivalent to that of $\mathrm{NaI}(\mathrm{Tl})[6]$, $\gamma$-ray spectroscopy applications become more feasible, especially when considering that the time constant of the scintillation light is fast enough (68-92 ns [7]) that higher rates can be accommodated before pulse pile up becomes an issue. It has also been reported that GAGG:Ce exhibits some degree of pulse shape discrimination (PSD) [7, 8, making identification of the type of incident radiation possible. However the peak emission wavelength, as shown in Table 1, is higher than most scintillators, so care must be taken when coupling GAGG scintillator to a SiPM to ensure a well-matched optical efficiency.

The focus of this paper is to exploit the high neutron-capture cross section of the gadolinium isotopes present in GAGG, combined with the high attenuation coefficient for efficient detection of neutron-induced $\gamma$ rays. These combined properties mean that GAGG has the potential to offer a very clean signature for 


\begin{tabular}{|c|c|c|c|c|c|c|}
\hline $\begin{array}{c}154 \mathrm{~Tb} \\
21.5 \mathrm{H} \\
\varepsilon: 100.00 \%\end{array}$ & $\begin{array}{c}15570 \\
5.320 \\
e: 100.00 \% \\
\beta<<0.10 \%\end{array}$ & $\begin{array}{c}156 \mathrm{~Tb} \\
5.35 \mathrm{D} \\
\varepsilon: 100.00 \%\end{array}$ & $\begin{array}{c}157 \mathrm{~b} \\
71 Y \\
E: 10.00 \%\end{array}$ & 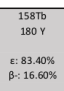 & $\begin{array}{l}\text { 1597b } \\
\text { STABLE } \\
100 \% \%\end{array}$ & $\begin{array}{c}160 \mathrm{~Tb} \\
72.3 \mathrm{D} \\
\beta=100.00 \% \%\end{array}$ \\
\hline 240.40 & $\begin{array}{l}\text { 15AAd } \\
\text { STABAE } \\
218 \%\end{array}$ & $\begin{array}{l}\text { STABIE } \\
14.80 \%\end{array}$ & $\begin{array}{l}\text { STABRE } \\
20.47 \%\end{array}$ & $\begin{array}{l}\text { STABI } \\
15.5 \%\end{array}$ & 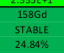 & \\
\hline E. 100.00\% & & & & & & $\beta=100.00 \%$ \\
\hline 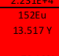 & 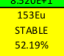 & $\begin{array}{l}5.074 \mathrm{EEA} \\
156 \mathrm{EU} \\
8.601 \mathrm{~V}\end{array}$ & $\begin{array}{l}1.1955 \\
155 \mathrm{u} \\
4.753 \mathrm{~V}\end{array}$ & $\begin{array}{l}156 \mathrm{U} \\
15.19 \mathrm{D}\end{array}$ & $\begin{array}{l}20202 \\
1577 \mathrm{u} \\
15.18 \mathrm{H}\end{array}$ & \\
\hline 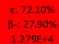 & 3.1225E+2 & $\begin{array}{l}\beta=99.98 \% \\
=: 0.02 \% \\
1=0.025 \%+3\end{array}$ & $\beta=100.00 \%$ & $\beta=100.00 \%$ & $\beta=100.00 \%$ & $\beta=100.00 \%$ \\
\hline $\begin{array}{l}\frac{1.299+4}{1515 m} \\
90 \%\end{array}$ & 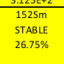 & $\begin{array}{l}1.35253 m \\
46.284 \mathrm{H} \\
\theta: 100.00 \%\end{array}$ & 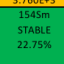 & 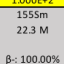 & $\begin{array}{l}1.10525-25 m \\
9.4 \mathrm{~m} \\
9: 100.00 \%\end{array}$ & $\begin{array}{l}1575 \mathrm{~m} \\
8.03 \mathrm{M} \\
8=100.00 \%\end{array}$ \\
\hline & $2.060 \mathrm{E}+2$ & $4.201 \mathrm{E}+2$ & 8323 & & & \\
\hline
\end{tabular}

Figure 1: Natural abundances and thermal neutron capture cross sections for the region around gadolinium (amended from NuDat 2.7[12]).

thermal neutron detection. Previous authors have reported the neutron detection performance of earlier gadolinium-containing scintillators, namely ceriumdoped gadolinium orthosilicate ( $\left.\mathrm{GSO}-\mathrm{Gd}_{2} \mathrm{SiO}_{5}: \mathrm{Ce}\right)[9$ and cerium-doped gadolinium iodide $\left(\mathrm{GdI}_{3}: \mathrm{Ce}\right)$ 10]. In particular, the performance of GSO as a thermal neutron detector was measured followed by a report on a prototype thin-GSO neutron detector 11 .

30 The gadolinium isotopes from mass 154 to 158 are stable, although only two isotopes, ${ }^{155} \mathrm{Gd}$ and ${ }^{157} \mathrm{Gd}$, have significant thermal neutron cross sections. The relevant section of the chart of the nuclides 12] is shown in Figure 1 with the respective abundances and $(n, \gamma)$ cross section displayed for each isotope. Both these gadolinium isotopes have extremely large thermal neutron capture cross sections and reasonably high natural abundances. ${ }^{155} \mathrm{Gd}$ has a natural abundance of $14.8 \%$ and a cross section of $60,740 \mathrm{~b}$ and makes a substantial contribution to the total neutron capture of gadolinium. However the majority of the response is from ${ }^{157} \mathrm{Gd}$ which has a natural abundance of $15.7 \%$ and a cross section of $253,700 \mathrm{~b}$. Overall, the effective $\sigma(n, \gamma)$ for natural gadolinium 40 is $49,000 \mathrm{~b}$.

The implication of the gadolinium thermal neutron capture cross section becomes apparent when the current status of thermal neutron detection technology is considered. The availability (and cost) of ${ }^{3} \mathrm{He}$ has been widely reported [1315. Alternatively, ${ }^{6} \mathrm{Li}$ and ${ }^{10} \mathrm{~B}[16,17]$ are both commonly used in detection 


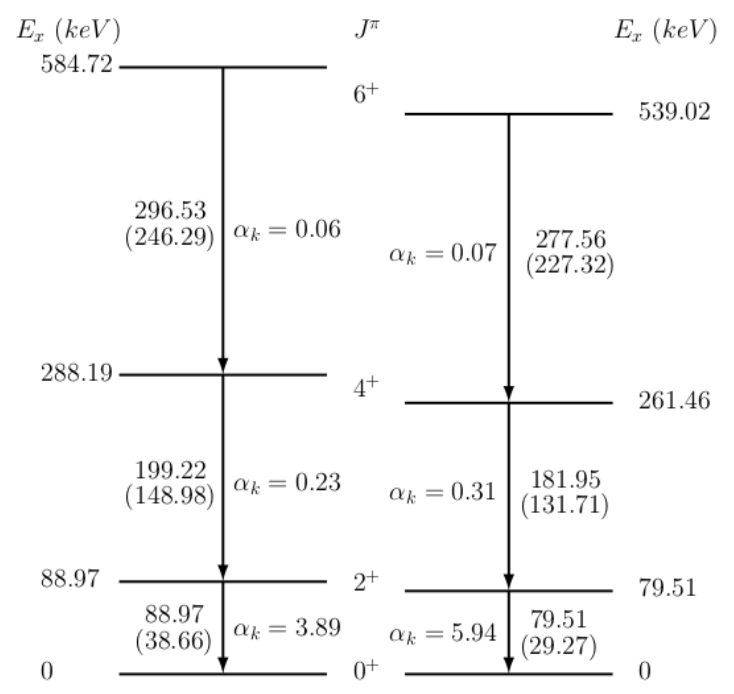

Figure 2: The low-lying level structure for the rotational bands of ${ }^{156} \mathrm{Gd}$ and ${ }^{158} \mathrm{Gd}$, adapted from [9, 22, 24]. The $\gamma$ ray transition energies are given alongside the competing internal conversion electron energy (in brackets).

${ }_{45}$ materials. Gaseous detectors using ${ }^{10} \mathrm{~B}$ are restricted to a relatively low pressure, and therefore low efficiency, but require a large detector volume[18, 19]. If ${ }^{6} \mathrm{Li}$ and ${ }^{10} \mathrm{~B}$ are loaded into scintillators the achievable loading fraction is limited by degradation of the scintillation characteristics 20, 21. In comparison, the thermal neutron capture cross section of gadolinium coupled with its mass fraction in GAGG means that relatively small crystals offer almost $100 \%$ thermal neutron capture efficiency.

The reaction products of $(n, \gamma)$ reactions on ${ }^{155} \mathrm{Gd}$ and ${ }^{157} \mathrm{Gd}$ are ${ }^{156} \mathrm{Gd}$ and ${ }^{158} \mathrm{Gd}$. The reaction Q-value energies are 8.536(2) and 7.937(2) MeV respectively. $(n, \gamma)$ reactions produce a large number of high energy $\gamma$ decays, typically above $1 \mathrm{MeV}$, which are not of primary interest in this work. The resulting deexcitations also produce transitions through numerous low-lying states, which are much more likely to be stopped within a relatively small detector crystal. The level scheme for the first three excited states in each of the nuclides of interest is given in Figure 2. The first excited states in ${ }^{156} \mathrm{Gd}$ and ${ }^{158} \mathrm{Gd}$ are at 88.97 


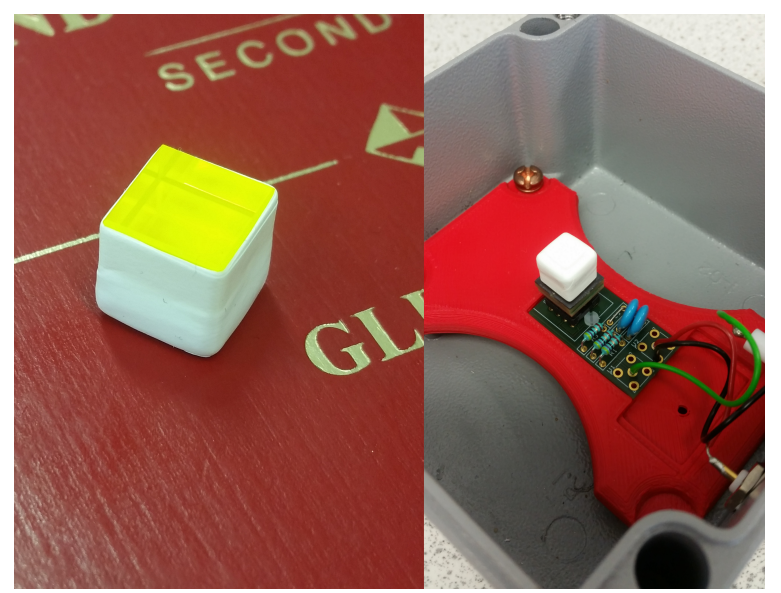

Figure 3: The $1 \mathrm{~cm}$ cube from Epic Crystal Co. and its mounting on the SensL J-series SiPM.

${ }_{60}$ and $79.51 \mathrm{keV}$ respectively, which are populated by E2 transitions of 199.22 and $181.95 \mathrm{keV}$ from the $4^{+}$2nd excited states. In turn the 2 nd excited states are themselves populated by $\mathrm{E} 2$ transitions of 296.4 and $277.6 \mathrm{keV}$ from the $6^{+} 3 \mathrm{rd}$ excited states. These $\gamma$ transitions are the source of key neutron signatures for gadolinium-containing scintillators. However, it should be noted that the $2^{+}$ first excited states in both isotopes can also decay via internal conversion processes, and this mechanism produces an additional low energy neutron-related peak due to the escape of characteristic gadolinium K-shell X-rays.

\section{Experimental Method}

\subsection{Scintillators}

Three samples of GAGG:Ce were acquired for this work: a smaller cube measuring $7.6 \mathrm{~mm}$ was provided by Advatech U.K. Ltd.; a second slightly larger $1 \mathrm{~cm}$ cube, and a thin slice $10 \mathrm{~mm} \times 10 \mathrm{~mm} \times 1 \mathrm{~mm}$ were both provided by Epic Crystal Co. Ltd. Both cubes were wrapped in PTFE tape to improve light collection; the $7.6 \mathrm{~mm}$ cube was only polished on a single face, whereas

75 the $1 \mathrm{~cm}$ cube had all faces polished, whilst the slice was polished on the two larger surfaces. 


\subsection{SensL SiPM}

The SiPM used in this work was a SensL J-series 2x2 array, model number ArrayJ-60035-4P-EVB. Each individual SiPM has an active area of $6.07 \mathrm{x}$ $6.07 \mathrm{~mm}^{2}$, which combined for a total package surface area of $155.25 \mathrm{~mm}^{2}$, with a total of 22,292 microcells per SiPM. The SiPM was readout by a bespoke breakout board which combined the four individual SiPM outputs into a single signal; the board and its mounting can be seen in Figure3. Each scintillator was optically coupled to the SiPM with Dow Corning high vacuum silicone grease 85 to ensure maximum light transmission.

\subsection{Data Acquisition and Pulse Height Analysis}

All $\gamma$ ray and neutron data were acquired with an Ortec EasyMCA and its accompanying Maestro software. The SiPM bias supply was set to $27.2 \mathrm{~V}$, or an overvoltage of $2.5 \mathrm{~V}$ as had previously been established for these devices 25.

The SiPM output signal was fed into a Canberra 2005 preamplifier and then an Ortec 672 spectroscopy amplifier with a shaping time of $2 \mu$ s and a gain of 10x.

The detectors were calibrated using the the primary photopeaks from the $\gamma$ sources ${ }^{241} \mathrm{Am},{ }^{57} \mathrm{Co},{ }^{22} \mathrm{Na}$, and ${ }^{137} \mathrm{Cs}$. Additionally, to improve the energy calibration in the low energy region around the first excited states of ${ }^{156} \mathrm{Gd}$

${ }_{95}$ and ${ }^{158} \mathrm{Gd}$, the $32.3 \mathrm{keV}$ barium X-ray peak from ${ }^{137} \mathrm{Cs}$ was also used in the calibration.

The thermal neutrons used in this work were produced from a $18.5 \mathrm{GBq}$ AmBe source located within a moderating water tank measuring $80 \mathrm{~cm} \times 76 \mathrm{~cm}$ x $94 \mathrm{~cm}$. The role of the water tank is to provide shielding from, and moderation of, the fast neutrons emitted from the $\operatorname{Be}(\alpha, n)$ reaction. The detector sits on top of the water tank, offset approximately $50 \mathrm{~cm}$ from the source, shielded by $35 \mathrm{~cm}$ of water. The dose rate immediately outside the water tank was monitored with a Berthold LB6411 Neutron Probe, which recorded a rate of $\sim 2 \mathrm{n} \mu \mathrm{Sv} / \mathrm{hr}$ throughout the experiment. In order to accurately determine the neutron sensitivity of our detector a complex shielding arrangement was necessary, shown in Figure 4. A dominant source of potential background is 


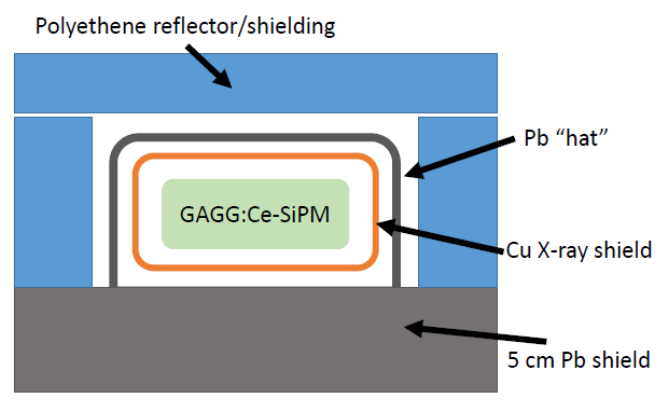

Figure 4: Sketch of the experimental setup sitting on top of the neutron tank. The AmBe source is located off the bottom of the figure surrounded by a water moderator.

the ${ }^{1} \mathrm{H}(\mathrm{n}, \gamma)^{2} \mathrm{D}$ reaction [26] from the water which causes a significant $\gamma$ flux. This is shielded from our detector by $5 \mathrm{~cm}$ of lead. In turn, the emission of characteristic lead X-rays from the shielding material must be removed using a $\sim 1 \mathrm{~mm}$ layer of copper wrapped around the detector. To avoid scattered $\gamma$ rays a further layer of lead $(\sim 3 \mathrm{~mm})$ was placed above the detector and to maximise neutron intensity a polyethene reflector $(2.5 \mathrm{~cm})$ was built around the whole assembly. Finally, additional layers of cadmium (a $3 \mathrm{~mm}$ thick sheet and a further $1 \mathrm{~mm}$ thick scattering "hat") could be put into place to remove thermal neutrons for background measurements.

\section{Results}

\subsection{Energy Resolution and Light Yield}

Pulse height spectra were collected using the calibration sources: ${ }^{137} \mathrm{Cs}$, ${ }^{22} \mathrm{Na}$, and ${ }^{241} \mathrm{Am}$, for each of the scintillator samples. The standard comparison of the ${ }^{137} \mathrm{Cs}$ photopeak is displayed in Figure 5 for each of the scintillator samples. Additionally, further data is displayed in Table 2 for the other calibration points. The finish of the non-coupled surfaces was different for each crystal so direct comparisons should be made with care. However, all samples achieve sub-10\% energy resolution at $661.7 \mathrm{keV}$, which is consistent with pre-

viously reported work on GAGG coupled to SiPM readout by Seitz et al[1]. 


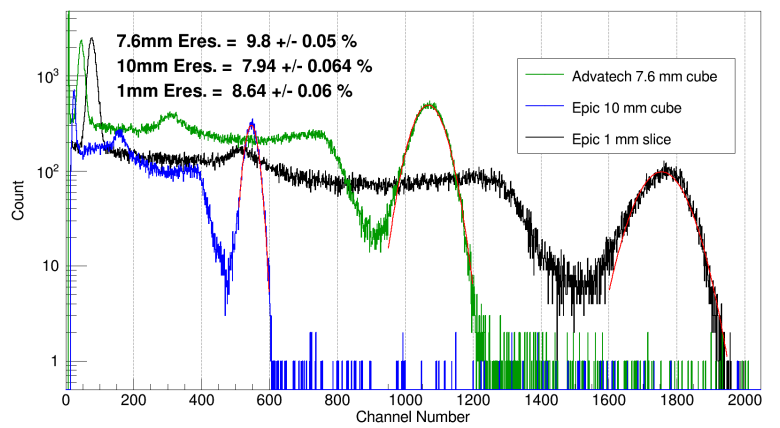

Figure 5: Uncalibrated ${ }^{137} \mathrm{Cs}$ spectra for each of the GAGG:Ce samples. Gaussian fits have been applied to the $661 \mathrm{keV}$ peaks and the respective energy resolutions have been extracted.

Furthermore, the exact composition of commercially available GAGG:Ce varies between suppliers, with dopant levels, growth technique, and elemental ratios contributing to the variability of quoted light yields ranging from 22,000-56,000 $\mathrm{pH} / \mathrm{MeV}$ 1, 7, 27, 29].

Figure 5 demonstrates the variable light yield obtained from our samples. In particular, by comparing the two samples from the same manufacturer, the effect of internal attenuation within the scintillator is clearly observed. The photopeak from the $10 \mathrm{~mm}$ thick scintillator occurs at a significantly lower channel number than the $1 \mathrm{~mm}$ sample, due to the internal absorption of the scintillation light 1 , $30-32$.

\subsection{AmBe Neutron Measurements}

The calibrated AmBe spectra for the $10 \mathrm{~mm}$ thick scintillator is shown in Figure 6 with several of the key features marked. The combined 1st excited state to ground state transitions for ${ }^{156} \mathrm{Gd}$ and ${ }^{158} \mathrm{Gd}$ at $\sim 82 \mathrm{keV}$ is the most prominent feature and is well separated from a lower energy peak at $\sim 43 \mathrm{keV}$. In addition two higher energy features are also observed at $\sim 259 \mathrm{keV}$ and $\sim 517 \mathrm{keV}$.

Similar features were observed in Ref. [9] and an explanation of their origins was initially presented there. The $82 \mathrm{keV}$ peak is the combination of the 88.9 and $79.5 \mathrm{keV} \gamma$ rays from the first excited states of ${ }^{156} \mathrm{Gd}$ and ${ }^{158} \mathrm{Gd}$, which 
Table 2: $\gamma$ ray photopeak energies for the calibration sources

\begin{tabular}{ccc}
\hline Sample & $E_{\text {peak }}(\mathrm{keV})$ & $E_{\text {res }}(\%)$ \\
\hline \multirow{4}{*}{$1 \mathrm{~mm}$} & 59.5 & $21.7 \pm 0.2$ \\
& 122 & $16.7 \pm 0.6$ \\
& 661 & $8.64 \pm 0.06$ \\
\hline \multirow{3}{*}{$7.6 \mathrm{~mm}$} & 59.5 & $25.3 \pm 0.07$ \\
& 511 & $10.3 \pm 0.1$ \\
& 661 & $9.80 \pm 0.05$ \\
\hline \multirow{3}{*}{$10 \mathrm{~mm}$} & 59.5 & $27.1 \pm 0.2$ \\
& 511 & $9.2 \pm 0.1$ \\
& 661 & $7.94 \pm 0.06$ \\
& 1274.5 & $5.6 \pm 0.3$ \\
\hline
\end{tabular}

145

cannot be separately resolved. The lower energy peak at $\sim 43 \mathrm{keV}$ is caused by internal conversion decays occurring within the GAGG scintillator from the first excited states. The internal conversion coefficients are both greater than 1 (3.89 and 5.94 respectively 24]) indicating de-excitation is predominantly via this mechanism. The thermal neutron capture cross sections of ${ }^{155} \mathrm{Gd}$ and ${ }^{157} \mathrm{Gd}$ are sufficiently large that the thermal neutron reaction occurs within tens of micrometers of the surface of the detector. Hence there is a high likelihood of escape by the $43 \mathrm{keV}$ gadolinium K-shell X-ray 33 for approximately half of the internal conversion events. These events produce the lower energy peak seen in Figure 6 due to the conversion electrons that only travel a few micrometres in the scintillator. Interestingly, the intensity of the lowest energy peak was far lower in [9] at $<50 \%$ than that of the 1st excited state transition, compared to $\sim 90 \%$ shown in this work. The gadolinium-containing scintillator discussed in [9], gadolinium orthosilicate (GSO), was measured to have an energy resolution of $9.6 \%$ at $661 \mathrm{keV}$ for a $1 \mathrm{~cm}$ cube coupled to a PMT. Reports of the fast neutron response of GAGG by Korzhik et al also report the presence of a neutron-induced $\gamma$ peak at $\sim 90 \mathrm{keV}[34$. 


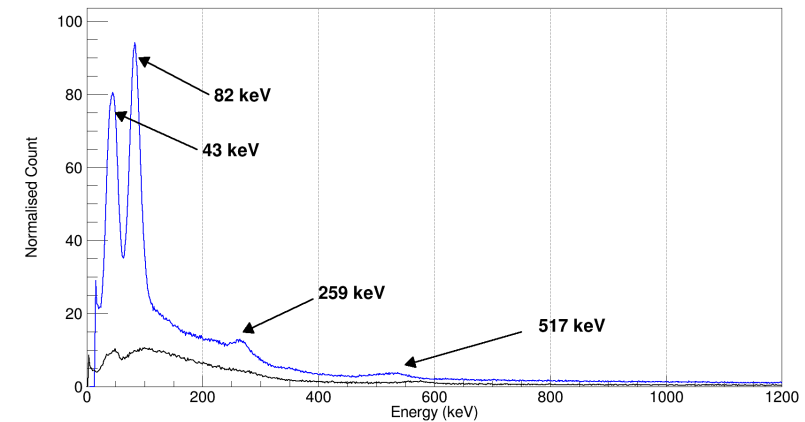

Figure 6: A typical $\gamma$-ray spectrum for thermal neutrons incident on a GAGG:Ce crystal (blue), and a background NaI:Tl spectrum (black).

In Figure 6 we also observe a $\gamma$ peak at $\sim 259 \mathrm{keV}$, this is a result of the rotational band structure of both ${ }^{156} \mathrm{Gd}$ and ${ }^{158} \mathrm{Gd}$ in which the $4^{+}$state de-excites in sum-coincidence through the $2^{+}$state to the ground state. The resulting $\gamma$ peak is due to the combination of these transitions from both ${ }^{156} \mathrm{Gd}$ and ${ }^{158} \mathrm{Gd}$ isotopes, and was also observed in [9].

The background $\gamma$-ray spectrum of the neutron tank as acquired with a $\mathrm{NaI}(\mathrm{Tl})$ detector is also displayed in Figure 6 which shows a shallow, broad peak at $\sim 560 \mathrm{keV}$ as well as a shoulder around $260 \mathrm{keV}$. There has been limited data published on the associated $\gamma$-ray spectra of isotopic neutron sources but AmBe and $\mathrm{PuBe}$ (the source discussed in [9]) have shown peaks at 0.2 and $0.5 \mathrm{MeV}[26$. Additionally, the thermal neutron capture cross section of NaI $\left({ }^{23} \mathrm{Na}: 0.43 \mathrm{~b}\right.$ and ${ }^{127} \mathrm{I}: 6.2 \mathrm{~b}$ ) results in a contribution from a $563 \mathrm{keV}$ transition in ${ }^{24} \mathrm{Na}$ and numerous $100<\mathrm{E}_{\gamma}<300 \mathrm{keV}$ decays from ${ }^{128}$ I, which will contribute to the 175 NaI spectrum in Figure 6

Since the majority of interactions are likely to be close to the surface of the material additional scintillator volume will not improve the neutron detection capability, but rather will have the opposite effect by increasing the sensitivity to background (and internal) $\gamma$ radiation. The effect of scintillator thickness is demonstrated in Figure 7 for the two pieces of scintillator from Epic Crystal Co. Ltd. The thinner scintillator no longer has the stopping power to fully 


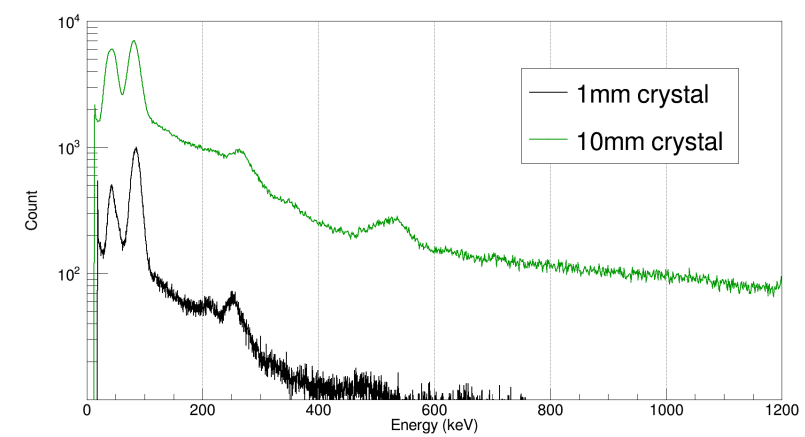

Figure 7: Neutron capture $\gamma$-ray spectra for GAGG:Ce scintillators of different thicknesses. The thinner scintillator has an improved peak-to-background ratio compared to the $10 \mathrm{~mm}$ thick sample.

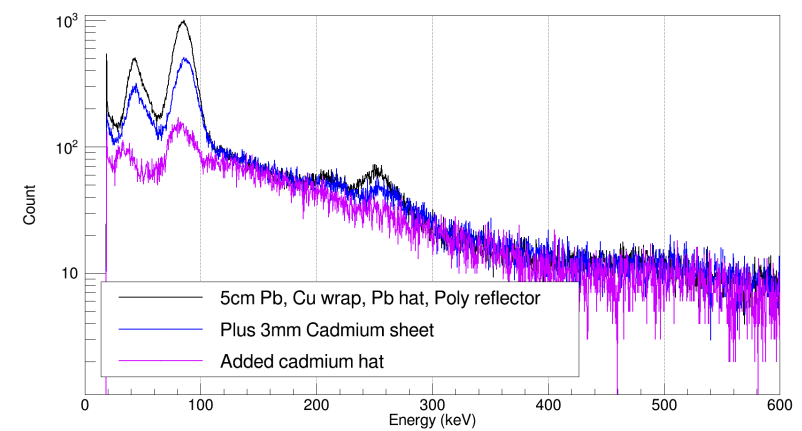

Figure 8: The response of GAGG:Ce's neutron capture capability to successive layers of cadmium shielding.

resolve the $\sim 517 \mathrm{keV}$ peak but the other features remain. The ratio of the $\sim 82 \mathrm{keV}$ peak to an extrapolated background is approximately $3.44 \pm 0.10$ for the $10 \mathrm{~mm}$ scintillator compared to $\sim 7.75 \pm 0.46$ for the thinner slice. Furthermore, measurements with the $1 \mathrm{~mm}$ sample recorded the best energy resolutions for the neutron peaks at $24.1 \pm 0.2 \%(\sim 82 \mathrm{keV})$ and $22.7 \pm 0.7 \%$ $(\sim 259 \mathrm{keV})$.

The impact of additional neutron shielding is displayed in Figure 8 by exploiting the large neutron resonances characteristic of cadmium. There is a 
$82 \mathrm{keV}$ peak-to-background ratio similarly reduces as thermal neutrons are captured prior to the detector, from $\sim 7.75$ to $3.98 \pm 0.29$ for the $\sim 3 \mathrm{~mm}$ cadmium layer between the source and detector, and a further reduction to $\sim 1.44 \pm 0.14$ once scattered neutrons are removed as well with the addition of a cadmium "hat".

\section{Conclusions}

We have confirmed the sensitivity of GAGG:Ce to thermal neutrons, and demonstrated the performance of a GAGG-based neutron detector using a SiPM-coupled detector. In particular, the two low energy peaks observed at $43 \mathrm{keV}$ and $82 \mathrm{keV}$ provide a clean signature for thermal neutron detection in GAGG, even in the presence of a strong $\gamma$ field. However, the intrinsic properties that make GAGG a viable candidate for spectroscopic photon applications such as PET, introduce potential limitations for exploitation as a neutron detector. For an effective neutron detector, the strong detection efficiency for $\gamma$ rays should be minimised by reducing the scintillator volume. This can be achieved without any significant reduction in neutron detection efficiency, and tends to reduce the sensitivity to unwanted high energy $\gamma$ rays and their associated Compton background. Smaller detector thicknesses also improve the overall light collection by minimising internal attenuation. The optimal limit for minimising the detector thickness is not yet known, but should be tested in terms of scintillator fabrication, retention of neutron response, and $\gamma$-ray insensitivity.

\section{Acknowledgments}

The authors would like to acknowledge the support of the STFC Nuclear

Davies, Henderson, and Smallcombe for their very patient conversations on nuclear structure. 


\section{References}

[1] B. Seitz, et al., Performance Evaluation of novel SiPM for Medical Imaging Applications, IEEE Tran. Nuc. Sci. 63 (2016) 503-508.

[2] A. G. Stewart, et al., Energy Resolution of Ce:GAGG and Pr:LuAG Scintillators Coupled to $3 \mathrm{~mm}$ x $3 \mathrm{~mm}$ Silicon Photomultipliers, IEEE Tran. Nuc. Sci. 63 (2016) 2496-2501.

[3] K. Nakanishi, S. Yamamoto, J. Kataoka, Performance comparison of finely pixelated LYSO- and GAGG-based Si-PM gamma cameras for high resolution SPECT, Nucl. Instr. Meth. A 872 (2017) 107-111.

[4] K. Shimazoe, et al., Double Photon Emission Coincidence Imaging using GAGG-SiPM pixel detectors, JINST 12 (2017) C12055.

[5] G. F. Knoll, Radiation Detection and Measurement, 3rd Edition, Wiley, 2000.

[6] V. V. Avdeichikov, et al., Light output and energy resolution of CsI, YAG, GSO, BGO, and LSO scintillators for light ions, Nucl. Instr. Meth. A 349 (1994) 216-224.

[7] H.-L. Kim, et al., Scintillation properties of the $\mathrm{Gd}_{3} \mathrm{Al}_{2} \mathrm{Ga}_{3} \mathrm{O}_{12}$ :Ce crystal, Journal of Ceramic Processing Research 16 (2015) 124-128.

[8] M. Kobayashi, et al., Significantly different pulse shapes for $\gamma$ - and $\alpha$-rays in $\mathrm{Gd}_{3} \mathrm{Al}_{2} \mathrm{Ga}_{3} \mathrm{O}_{12}$ :Ce3+ scintillating crystals, Nucl. Instr. Meth. A 694 (2012) 91-94.

[9] P. L. Reeder, Neutron detection using GSO scintillator, Nucl. Instr. Meth. A 340 (1994) 371-378.

[10] J. Glodo, W. M. Higgins, E. V. D. van Loef, K. S. Shah, GdI $_{3}:$ Ce - A New Gamma and Neutron Scintillator, in: IEEE Nuclear Science Symposium Conference Record, 2006. 
[11] P. L. Reeder, Thin GSO scintillator for neutron detection, Nucl. Instr. Meth. A 353 (1994) 134-136.

[12] Nudat 2.7, https://www.nndc.bnl.gov, accessed October 2018.

[13] R. T. Kouzes, The ${ }^{3}$ He supply problem, Tech. Rep. PNNL-18388, Pacific Northwest National Laboratory (2009).

[14] M. L. Wald, Shortage Slows a Program to Detect Nuclear Bombs, The New York Times (2009) A12.

[15] R. T. Kouzes, A. T. Lintereur, E. R. Siciliano, Progress in alternative neutron detection to address the helium-3 shortage, Nucl. Instr. Meth. A 784 (2015) 172-175.

[16] E. Segrè, C. Wiegarnd, Boron trifluoride neutron detector for low neutron intensities, Rev. Sci. Instrum. 18 (1947) 86-89.

[17] R. Stedman, Scintillator for thermal neutrons using $\mathrm{Li}^{6} \mathrm{~F}$ and $\mathrm{ZnS}(\mathrm{Ag})$, Rev. Sci. Instrum. 31 (1960) 1156.

[18] J. H. Ely, E. R. Siciliano, A. T. Lintereur, M. T. Swinhoe, Alternatives for Helium-3 in Multiplicity Counters, IEEE Trans. Nucl. Sci. 60 (2013) $510-514$.

[19] A. Lintereur, et al., ${ }^{3} \mathrm{He}$ and $\mathrm{BF}_{3}$ neutron detector pressure effect and model comparison, Nucl. Instr. Meth. A 652 (2011) 347-350.

[20] D. M. Drake, W. C. Feldman, C. Hurlbut, New Elecetronically Black Neutron Detectors, Nucl. Instr. Meth. A 247 (1986) 576-582.

[21] N. J. Cherepy, et al., Bismuth- and lithium-loaded plastic scintillators for gamma and neutron detection, Nucl. Instr. Meth. A 778 (2015) 126-132.

[22] C. W. Reich, Nuclear Data Sheets for A = 156, Nuclear Data Sheets 113 (2012) 2537-2840. 
[23] N. Nica, Nuclear Data Sheets for A=158, Nuclear Data Sheets 141 (2017)

[24] T. Kibédi, et al., Evaluation of theoretical conversion coefficients using BrIcc, Nucl. Instr. Meth. A 589 (2008) 202-229.

[25] M. P. Taggart, P. J. Sellin, Comparison of the pulse shape discrimination performance of plastic scintillators coupled to a SiPM, Nucl. Instr. Meth.

[26] H. R. Vega-Carillo, E. Manzanares-Acuña, A. M. Becerra-Ferreiro, A. Carrilo-Nuñez, Neutron and gamma ray spectra of ${ }^{239} \mathrm{PuBe}$ and ${ }^{241} \mathrm{AmBe}$, Applied Radiation and Isotopes 57 (2002) 167-170.

[27] S. Rawat, et al., Pulse shape discrimination properties of

[32] K. Kamada, et al., 2 inch diameter single crystal growth and scintillation properties of Ce: $\mathrm{Gd}_{3} \mathrm{Al}_{2} \mathrm{Ga}_{3} \mathrm{O}_{12}$, J. Cryst. Growth 352 (2012) 88-90. 
[33] D. Schultz, et al., The K-shell Auger electron spectrum of gadolinium obtained using neutron capture in a solid state device, J. Phys. D: Appl. Phys. 43 (2010) 075502.

[34] M. Korzhik, et al., Compact and effective detector of the fast neutrons on a base of Ce doped $\mathrm{Gd}_{3} \mathrm{Al}_{2} \mathrm{Ga}_{3} \mathrm{O}_{12}$ scintillation crystal, IEEE Trans. Nucl. Sci.doi:10.1109/TNS.2018.2888495. 